\title{
Malária em região extra-Amazônica: situação no Estado de Santa Catarina
}

\author{
Malaria outside the Brazilian Amazonian region: \\ the situation in Santa Catarina State
}

\section{Ricardo Luiz Dantas Machado', Álvaro Augusto Ribeiro D’ Almeida Couto², Carlos Eugênio Cavasini' e Vanja Sueli Pachiano Calvosa ${ }^{3}$}

\begin{abstract}
Resumo Este estudo objetiva conhecer o perfil epidemiológico da malária no Estado de Santa Catarina, analisando dados disponibilizados pela Fundação Nacional de Saúde, relativos ao período de 1996/2001. Das 4.707 lâminas examinadas, 5,5\% evidenciaram-se positivas. As infecções por Plasmodium vivax foram 69\%, por Plasmodium falciparum $25,6 \%$, infecções mistas por ambos foram $5 \%$ e, somente $0,4 \%$ por Plasmodium malariae. Foi observado $67,4 \%$ casos importados e 32,6\% casos autóctones. Nos últimos anos houve um aumento de casos importados. A maioria destes veio da região Amazônica brasileira e o restante de países africanos. Identificou-se os municípios de Joinville, Blumenau, São Francisco do Sul e Florianópolis com maior número de autoctonia no biênio 1996/97. Medidas de controle e vigilância fazem-se necessárias, no sentido de prevenir a reintrodução do plasmódio, favorecendo a autoctonia. Será útil o mapeamento das áreas de risco, já que é contínua a expectativa de sua reemergência em áreas hoje consideradas sob controle.
\end{abstract}

Palavras-chaves: Malária. Epidemiologia. Estado de Santa Catarina. Brasil.

Abstract This study aimed to evaluate the malaria epidemiological aspects in Santa Catarina State, Brazil, by using National Health Foundation data from 1996 to 2001. From 4,707 thick smears analyzed 5.5\% were positive. Plasmodium vivax was found in $69.0 \%$; Plasmodium falciparum in $25.6 \%$, mixed infection with both in $5 \%$, and Plasmodium malariae in only $0.4 \%$. It was observed that $67.4 \%$ were heterochthonous cases and $32.6 \%$ autochthonous cases. In recent years, the incidence of heterochthonous cases has increased. The majority of these cases come from the Brazilian Amazon region and the remainder from African countries. However, the municipalities of Joinville. Blumenau. São Francisco do Sul and Florianópolis registered higher rates of autochthonous cases in 1996/1997. Control and epidemiological surveillance are necessary to prevent the reintroduction of Plasmodium in this region. It would be useful to investigate each epidemiological setting in order to prevent the reappearance of the disease in areas currently considered under control.

Key-words: Malaria. Epidemiology. Santa Catarina State. Brazil.

O impacto global referente aos danos sócioeconômicos causados pela malária é extenso, representando essa doença um dos maiores problemas de saúde pública nas áreas tropicais e subtropicais do mundo ${ }^{16}$. A incidência da malária no Brasil aumentou cerca de dez vezes nos últimos 30 anos e, atualmente mais de $99 \%$ dos casos ocorrem na região Amazônica9. A malária é uma doença infecciosa aguda, causada por protozoários do gênero Plasmodium. Os agentes etiológicos da malária presentes no Brasil são das espécies Plasmodium vivax, Plasmodium falciparum e Plasmodium malariae e a transmissão ocorre através da picada de mosquitos fêmeas do gênero Anopheles, apesar de outros mecanismos serem incriminados em determinadas situações.

A dinâmica de transmissão da malária é variável entre os grupamentos humanos pertencentes a distintos estratos epidemiológicos, observando-se a

\footnotetext{
1. Departamento de Doenças Dermatológicas, Infecciosas e Parasitárias da Faculdade de Medicina de São José do Rio Preto, São José do Rio Preto, SP. 2. Centro de Referência de Doenças Tropicais da Secretaria de Saúde do Estado do Amapá, Macapá, AP. 3. Seção de Parasitologia do Instituto Evandro Chagas, Secretaria de Vigilância em Saúde do Ministério da Saúde, Belém, PA.

Endereço para correspondência: Dr. Ricardo Luiz Dantas Machado. Centro de Investigação de Microrganismos. Av. Brigadeiro Faria Lima 5416, 15090-000 São José do Rio Preto, SP.

Tel: 55172105700 ramal 5887

e-mail: ricardomachado@famerp.br

Recebido para publicação em 3/10/2002

Aceito em 8/8/2003
} 
interação de fatores de risco de origens diferentes, tais como de ordem biológica, e ecológica, econômica, socioculturais e de saúde pública ${ }^{15}$. A associação desses fatores determina diferentes patamares de transmissão que originam níveis diferenciados na prevalência da endemia ${ }^{8}$.

A dispersão da malária no Brasil assumiu maior importância epidemiológica a partir da década de 60, quando foram construídas importantes rodovias unindo a região Norte às outras regiões ${ }^{12}$. A partir dos anos 70 e até o final dos anos 80 , houve um aumento do número de casos de malária registrados nesta região, oportunidade em que foram evidenciadas altas taxas de crescimento demográfico, influenciadas, principalmente, pela construção de novas rodovias, aberturas de projetos de colonização e expansão de áreas de garimpos. Esta migração desordenada à Amazônia foi considerada como determinante para o aumento quantitativo de casos e a expansão territorial da malária na região ${ }^{12}$. O Estado de Rondônia foi o que recebeu o maior número de migrantes, motivados pelos projetos de assentamentos coordenados pelo Instituto Nacional de Colonização e Reforma Agrária (INCRA), sendo estes fatores responsáveis pelo crescimento abrupto de sua população ${ }^{11}$. O fluxo populacional para os Estados do Pará e Mato Grosso originou-se da região Nordeste do país, particularmente do Estado do Maranhão. No sudeste do Pará, a descoberta de garimpos levou a uma elevação no número de migrantes das áreas rurais, provenientes principalmente, dos Estados do Maranhão, Piauí, Goiás e Ceará ${ }^{12}$. Durante a década de 80 as populações de Rondônia e Roraima praticamente dobraram seus contingentes populacionais, já no Acre, os projetos de assentamento levaram a um aumento demográfico de quase sete vezes no ano de 1985. A partir daí, a movimentação populacional estabelecida intra e extra Amazônia Legal, determinou forte influência nas áreas de alta transmissão de malária, não somente para as micro regiões mais próximas, como também para localidades mais distantes da Hiléia
Amazônica, chegando a áreas fora da região Amazônica $^{12}$. A malária no Brasil apresentou-se ascendente até 1989 , atingindo mais de 570.000 casos diagnosticados. Posteriormente, verificou-se a estabilização deste número em níveis elevados, freqüentemente acima de 500mil casos/ano, e no biênio 1996/97 observou-se uma queda acentuada do número de $\operatorname{casos}^{13}$. Este fato começou a ser observado com a implementação da estratégia de controle da malária definida a partir da reunião ministerial promovida pela OMS em Amsterdã (1992), que por meio de diagnóstico e tratamento precoces, detecção pronta de epidemias e planejamento e aplicação de medidas antivetoriais seletivas, visava impedir a mortalidade e a redução da morbidade e as perdas sociais e econômicas devido à malária. Associadas a isto, a redução de deslocamentos para a área endêmica em virtude das dificuldades econômicas, o esgotamento das áreas de extração mineral, a estabilidade da população em projetos agrícolas e a utilização de esquemas terapêuticos de maior eficácia, também contribuíram para aquela redução ${ }^{10}$.

$\mathrm{Na}$ última década, foram registrados vários focos de malária fora da região Amazônica causados pela presença de indivíduos infectados em áreas nas quais a densidade anofélica é verdadeiramente alta para o restabelecimento do ciclo. A dimensão desses surtos, posterior à introdução desses casos importados, oscila diretamente com o sistema de vigilância epidemiológica empregado e, indiretamente com o desenvolvimento sócio-econômico da região ${ }^{2}$. No Estado de Santa Catarina os estudos epidemiológicos são raros, restritos apenas àqueles realizados pelas entidades responsáveis pelo controle da malária no país. Alguns estudos foram realizados na década de $60^{5714}$ e o último estudo avaliando o envolvimento de Anopheles (Kertezia) na transmissão desta doença, data de $1984^{6}$.

Este estudo tem como objetivo conhecer o perfil epidemiológico da malária no Estado de Santa Catarina, analisando-se os dados disponibilizados pela Fundação Nacional de Saúde/Gerência Técnica de Malária, relativos ao período de 1996 a 2001.

\section{MATERIAL E MÉTODOS}

O estudo foi realizado a partir dos dados extraídos dos relatórios de casos de malária elaborados pelo Ministério da Saúde/Fundação Nacional de Saúde/ Centro Nacional de Epidemiologia/Gerência Técnica de Malária (MS/FUNASA/CENEPI/GTMAL), no período de janeiro de 1996 a dezembro de 2001. Deste material, colheram-se dados referentes ao número de lâminas examinadas para a pesquisa do parasito, número de lâminas positivas de acordo com a espécie de Plasmodium e a origem dos pacientes positivos.

As informações foram consolidadas em tabelas, objetivando reunir dados específicos aos interesses do estudo para análise e avaliação dos fenômenos epidemiológicos de maior relevância.

\section{RESULTADOS}

No período considerado para a realização dessa investigação, foram efetuadas 4.707 lâminas para a pesquisa de plasmódios, das quais 258 foram consideradas positivas pelo método da gota espessa, evidenciando-se uma dimensão de positividade de $5,5 \%$ (Tabela 1 ).
Conforme os dados mostrados na Tabela 2, registraramse 258 casos positivos. As infecções causadas por Plasmodium vivax foram 178 (69\%), por Plasmodium falciparum 66 (25,6\%), com infecções mistas, por associação de $P$. falciparum e o P. vivax foram $13(5 \%)$ e, finalmente, apenas $1(0,4 \%)$ por Plasmodium malariae. 
Foi observado um total de 84 (32,6\%) casos autóctones e de $174(67,4 \%)$ casos importados durante o período estudado. No primeiro ano da série, em 1996, todos os casos registrados de malária foram autóctones. A partir de 1997 evidenciou-se o aumento (32,3\%) de casos importados de malária. De 1998 a 2001, foram registrados $100 \%, 96,3 \%, 98 \%$ e $100 \%$ de casos importados, respectivamente (Tabela 3). Considerando os dados constantes da Tabela 4, onde os casos autóctones registrados no período estudado foram distribuídos por localidade de ocorrência, identificaram-se os municípios com maior número de autoctonia, particularmente no biênio 1996/1997. Destacam-se nesta situação os municípios de Joinville com 17

\begin{tabular}{|c|c|c|c|}
\hline \multirow{3}{*}{ Ano } & \multicolumn{3}{|c|}{ Lâminas } \\
\hline & \multirow{2}{*}{$\begin{array}{c}\text { examinadas } \\
n^{\circ}\end{array}$} & \multicolumn{2}{|c|}{ positivas } \\
\hline & & $\mathrm{n}^{\circ}$ & $\%$ \\
\hline 1996 & 1.178 & 59 & 5,0 \\
\hline 1997 & 928 & 34 & 3,7 \\
\hline 1998 & 1.536 & 46 & 3,0 \\
\hline 1999 & 463 & 27 & 5,8 \\
\hline 2000 & 560 & 50 & 8,9 \\
\hline 2001 & 42 & 42 & 100,0 \\
\hline Total & 4.707 & 258 & 5,5 \\
\hline
\end{tabular}

Fonte: MS/FUNASA/CENEP/GTM, 2002

\begin{tabular}{|c|c|c|c|c|c|c|c|c|c|}
\hline \multirow[t]{2}{*}{ Ano } & \multirow{2}{*}{$\begin{array}{c}\text { Lâminas } \\
\text { positivas } \\
n^{\circ}\end{array}$} & \multicolumn{2}{|c|}{$\begin{array}{c}\text { Plasmodium } \\
\text { vivax }\end{array}$} & \multicolumn{2}{|c|}{$\begin{array}{l}\text { Plasmodium } \\
\text { falciparum }\end{array}$} & \multicolumn{2}{|c|}{$\begin{array}{c}\text { Plasmodium } \\
\text { malariae }\end{array}$} & \multicolumn{2}{|c|}{$\begin{array}{c}\text { P. vivax }+ \\
\text { P. falciparum }\end{array}$} \\
\hline & & $\mathrm{n}^{\circ}$ & $\%$ & $\mathrm{n}^{\circ}$ & $\%$ & $\mathrm{n}^{\circ}$ & $\%$ & $\mathrm{n}^{\circ}$ & $\%$ \\
\hline 1996 & 59 & 37 & 62,7 & 17 & 28,8 & - & - & 5 & 8,5 \\
\hline 1997 & 34 & 23 & 67,6 & 10 & 29,4 & - & - & 1 & 3,0 \\
\hline 1998 & 46 & 32 & 69,6 & 13 & 28,3 & - & - & 1 & \\
\hline 1999 & 27 & 20 & 74,1 & 7 & 25,9 & - & - & - & - \\
\hline 2000 & 50 & 32 & 64,0 & 14 & 28,0 & 1 & 2,0 & 3 & 6,0 \\
\hline 2001 & 42 & 34 & 81,0 & 5 & 11,9 & - & - & 3 & 7,1 \\
\hline Total & 258 & 178 & 69,0 & 66 & 25,6 & 1 & 0,4 & 13 & 5,0 \\
\hline
\end{tabular}

Fonte: MS/FUNASA/CENEP/GTM, 2002

Tabela 3 - Casos de malária detectados no Estado de Santa Catarina, de acordo com a autoctonia, no período de 1996 a 2001.

\begin{tabular}{lcrrrrrr}
\hline Ano & Lâminas positivas & & \multicolumn{2}{c}{ Casos autóctones } & & \multicolumn{2}{c}{ Casos importados } \\
\cline { 3 - 4 } \cline { 7 - 8 } & $\mathrm{n}^{\circ}$ & 59 & 100,0 & & 0 & - \\
\hline 1996 & 59 & 23 & 67,6 & & 11 & 32,3 \\
1997 & 34 & 0 & - & & 46 & 100,0 \\
1998 & 46 & 1 & 3,7 & & 26 & 96,3 \\
1999 & 27 & 1 & 2,0 & & 49 & 98,0 \\
2000 & 50 & 0 & - & & 42 & 100,0 \\
2001 & 42 & 84 & 32,6 & & 174 & 67,4
\end{tabular}

Fonte: MS/FUNASA/CENEP/GTM, 2002

ocorrências, Blumenau e São Francisco do Sul com oito cada e Florianópolis com seis registros.

A maioria dos casos importados originou-se da região Amazônica brasileira num total de 146 (84\%), 2 (1\%) casos de estados do Nordeste brasileiro e o restante 26 (15\%) de países africanos. A participação dos Estados do Pará e Rondônia foi significativa, contribuindo com 44 (25,3\%) cada um, e a de Mato Grosso com 23 (13,2\%), sendo estes os responsáveis pela maior demanda de malária para o Estado de Santa Catarina (Tabela 5). 
Tabela 4 - Autoctonia de malária no Estado de Santa Catarina, de acordo com os municípios, no período de 1996 a 2001.

\begin{tabular}{|c|c|c|c|c|c|c|}
\hline & 1996 & 1997 & 1998 & 1999 & 2000 & 2001 \\
\hline Municípios & & & & & & \\
\hline Alfredo Wagner & 0 & 1 & 0 & 0 & 0 & 0 \\
\hline Araquari & 0 & 1 & 0 & 0 & 0 & 0 \\
\hline Araranguá & 0 & 1 & 0 & 0 & 0 & 0 \\
\hline Blumenau & 8 & 1 & 0 & 0 & 0 & 0 \\
\hline Caibi & 0 & 1 & 0 & 0 & 0 & 0 \\
\hline Camboriu & 1 & 0 & 0 & 0 & 0 & 0 \\
\hline Canoinhas & 2 & 1 & 0 & 0 & 0 & 0 \\
\hline Chapecó & 1 & 1 & 0 & 0 & 0 & 0 \\
\hline Criciúma & 2 & 0 & 0 & 0 & 0 & 0 \\
\hline Cunha Pora & 0 & 1 & 0 & 0 & 0 & 0 \\
\hline Florianópolis & 6 & 1 & 0 & 0 & 0 & 0 \\
\hline Gaspar & 0 & 1 & 0 & 0 & 0 & 0 \\
\hline Içara & 0 & 1 & 0 & 0 & 0 & 0 \\
\hline Ilhota & 0 & 1 & 0 & 0 & 0 & 0 \\
\hline Indaial & 0 & 0 & 0 & 1 & 0 & 0 \\
\hline Itajaí & 5 & 1 & 0 & 0 & 0 & 0 \\
\hline Joinville & 17 & 2 & 0 & 0 & 0 & 0 \\
\hline Lages & 2 & 0 & 0 & 0 & 0 & 0 \\
\hline Mafra & 0 & 2 & 0 & 0 & 0 & 0 \\
\hline Orleans & 2 & 0 & 0 & 0 & 0 & 0 \\
\hline Porto União & 0 & 2 & 0 & 0 & 0 & 0 \\
\hline Riqueza & 1 & 0 & 0 & 0 & 0 & 0 \\
\hline Rodeio & 0 & 0 & 0 & 0 & 1 & 0 \\
\hline Santo Amaro da Imperatriz & 1 & 0 & 0 & 0 & 0 & 0 \\
\hline São Francisco do Sul & 8 & 2 & 0 & 0 & 0 & 0 \\
\hline São João Batista & 2 & 0 & 0 & 0 & 0 & 0 \\
\hline São José & 0 & 1 & 0 & 0 & 0 & 0 \\
\hline Schroeder & 1 & 0 & 0 & 0 & 0 & 0 \\
\hline Urussanga & 0 & 1 & 0 & 0 & 0 & 0 \\
\hline Total & 59 & 23 & 0 & 1 & 1 & 0 \\
\hline
\end{tabular}

Fonte: MS/FUNASA/CENEP/GTM, 2002

Tabela 5 - Casos importados de malária no Estado de Santa Catarina no período de 1996 a 2001.

\begin{tabular}{|c|c|c|c|c|c|c|c|c|c|c|c|c|c|c|}
\hline \multirow{3}{*}{$\begin{array}{l}\text { Origem dos casos } \\
\text { importados }\end{array}$} & \multirow{2}{*}{\multicolumn{2}{|c|}{$\begin{array}{c}\text { Total } \\
\text { de casos }\end{array}$}} & \multicolumn{10}{|c|}{ Ano } & \multirow{2}{*}{\multicolumn{2}{|c|}{2001}} \\
\hline & & & \multicolumn{2}{|c|}{1996} & \multicolumn{2}{|c|}{1997} & \multicolumn{2}{|c|}{1998} & \multicolumn{2}{|c|}{1999} & \multicolumn{2}{|c|}{2000} & & \\
\hline & $\mathrm{n}^{\circ}$ & $\%$ & $\mathrm{n}^{\circ}$ & $\%$ & $\mathrm{n}^{\circ}$ & $\%$ & $\mathrm{n}^{\circ}$ & $\%$ & $\mathrm{n}^{\circ}$ & $\%$ & $\mathrm{n}^{\circ}$ & $\%$ & $\mathrm{n}^{\circ}$ & $\%$ \\
\hline Acre & 1 & 0,6 & 0 & - & 0 & - & 0 & - & 0 & - & 1 & 100,0 & 0 & - \\
\hline Amapá & 11 & 6,3 & 0 & - & 0 & - & 0 & - & 0 & - & 5 & 45,5 & 6 & 54,5 \\
\hline Amazonas & 8 & 4,6 & 0 & - & 2 & 25,0 & 1 & 12,5 & 1 & 12,5 & 3 & 37,5 & 1 & 12,5 \\
\hline Maranhão & 3 & 1,7 & 0 & - & 0 & - & 1 & 33,3 & 0 & - & 1 & 33,3 & 1 & 33,3 \\
\hline Mato Grosso & 23 & 13,2 & 0 & - & 1 & 4,3 & 4 & 17,4 & 4 & 17,4 & 10 & 43,5 & 4 & 17,4 \\
\hline Mato Grosso do Sul & 2 & 1,2 & 0 & - & 0 & - & 2 & 100,0 & 0 & - & 0 & - & 0 & - \\
\hline Pará & 44 & 25,3 & 0 & - & 1 & 2,3 & 10 & 22,7 & 7 & 15,9 & 12 & 27,3 & 14 & 31,8 \\
\hline Pernambuco & 1 & 0,6 & 0 & - & 0 & - & 0 & - & 0 & - & 0 & - & 1 & 100,0 \\
\hline Piauí & 1 & 0,6 & 0 & - & 0 & - & 0 & - & 0 & - & 0 & - & 1 & 100,0 \\
\hline Rondônia & 44 & 25,3 & 0 & - & 5 & 11,4 & 19 & 43,1 & 8 & 18,2 & 7 & 15,9 & 5 & 11,4 \\
\hline Roraima & 1 & 0,6 & 0 & - & 0 & - & 1 & 100 & 0 & - & 0 & - & 0 & - \\
\hline Ignorados & 9 & 5,2 & 0 & - & 0 & - & 0 & - & 0 & - & 2 & 22,2 & 7 & 77,8 \\
\hline Países Africanos & 26 & 14,8 & 0 & - & 2 & 7,7 & 8 & 30,8 & 6 & 23,0 & 8 & 30,8 & 2 & 7,7 \\
\hline Total & 174 & 100,0 & 0 & - & 11 & 6,3 & 46 & 26,4 & 26 & 14,9 & 49 & 28,2 & 42 & 24,2 \\
\hline
\end{tabular}

Fonte: MS/FUNASA/CENEP/GTM, 2002 


\section{DISCUSSÃO}

Nas décadas de 80 e 90, grandes avanços foram alcançados na luta contra a malária, e atualmente observa-se que a doença está praticamente restrita à região Amazônica, onde registram-se mais de 99\% dos casos. Segundo avaliação do programa nacional de controle da malária, em 1999, foi registrado um aumento da incidência da doença que chegou a atingir níveis insustentáveis, colocando em risco o desenvolvimento da região Amazônica. Neste ano, foram registrados 635.644 casos de maláriå. Em 2000, 2001 e 2002 houve redução da incidência.

Deve-se atentar para o fato de que a fragilidade no processo de vigilância e controle da malária dentro da Amazônia pode levar a disseminação da doença novamente para as outras áreas do país, como observado na década de 80 . A migração humana é uma realidade entre as diferentes regiões do país, e a pouca habilidade no manejo clínico dos diversos profissionais da área de saúde, bem como a falta de locais disponíveis para o diagnóstico em áreas não endêmicas se constitui em um sério agravante para a saúde pública nestas regiões.

No Estado de Santa Catarina, através da avaliação dos relatórios de casos suspeitos de malária realizado pela Ministério da Saúde/Fundação Nacional de Saúde/ Centro Nacional de Epidemiologia/Gerência Técnica de Malária, observa-se um decréscimo no número de casos autóctones de malária nos últimos cinco anos (Tabela 1 e 2), entretanto, denota-se também, um aumento de casos importados da doença (Tabela 3).

Os casos autóctones registrados durante os anos de 1996 e 1997 concentraram-se principalmente, nos municípios localizados ao norte e nordeste do Estado; localidades com grande potencial turístico e industrial, fato este que atrai pessoas de outras regiões em busca de lazer e emprego. Além disso, o município de São Francisco do Sul está localizado em uma área portuária, servindo como porta de entrada para pessoas oriundas de outras localidades do país e do mundo. Apesar de observarmos que a maioria dos casos importados de malária nos últimos anos, tiveram origem principalmente dos Estados do Pará, Rondônia e Mato Grosso, verificouse também a contribuição de outras áreas da Amazônica brasileira, e de alguns países africanos (Tabela 5). Devese pensar, entretanto, que o fato de se ter encontrado poucos casos, não significa a possibilidade de não ocorrer malária no estado, pois casos de portadores assintomáticos do plasmódio (PAP), não diagnosticados e sem tratamento, já foram registrados em áreas da mata Atlântica, o que pode possibilitar a manutenção do parasito na região ${ }^{3}$. Associado a este fato, está o desconhecimento das atuais espécies vetoras que estão eventualmente circulando nestas localidades e que podem contribuir para um possível surto da doença.

Para a área extra-amazônica, como é o caso do Estado de Santa Catarina, é necessário que as atividades de vigilância epidemiológica sejam desenvolvidas continuamente, avaliando a sua vulnerabilidade e suscetibilidade nas diferentes regiões, e viabilizando o diagnóstico rápido e tratamento em toda a rede de serviços de saúde ${ }^{1}$. Além disso, será útil o mapeamento das áreas de risco, bem como a reavaliação das espécies vetoras potencialmente incriminadas na transmissão, já que é contínua a expectativa da reintrodução do plasmódio e reemergência da malária em áreas hoje consideradas sob controle.

\section{AGRADECIMENTOS}

Ao Ministério da Saúde/Fundação Nacional de Saúde/Centro Nacional de Epidemiologia/Gerência Técnica de Malária pelo fornecimento dos dados para o objetivo deste estudo.

\section{REFERÊNCIAS BIBLIOGRÁFICAS}

1. Albuquerque $B C$. Malária Urbana em áreas endêmicas e não endêmicas. In: Resumos da Reunião Nacional dos Pesquisadores de Malária: Conselho Nacional de Desenvolvimento Científico Tecnológico, Fundação Nacional Saúde, RONET, Pan American Health Organization/World Health Organization. Porto Velho p. 57, 2002.

2. Barata RC. Malária no Brasil: Panorama Epidemiológico na última década. Caderno de Saúde Pública 11: 128-136,1995.

3. Barroso WJ. Brasil: A Malária na Mata Atlântica. Revista do Conselho Federal de Medicina 136: 15-16, 2002.

4. Cavasini MTV, Ribeiro WL, Kawamoto F, Ferreira MU. How prevalent is Plasmodium malariae in Rondônia, Western Brazilian Amazon? Revista da Sociedade Brasileira de Medicina Tropical 33: 489-492, 2000.

5. Coutinho JO, Rachou R, Ferreira M. Considerations concerning a preliminary inspection of malaria in a zone of high endemicity in the State of Santa Catarina. Revista Brasileira de Malariologia e Doenças Tropicais 18: 533-551, 1966.

6. Deane LM, Ferreira Neto J, Lima MM. The vertical dispersion of Anopheles (Kertezia) cruzi in a forest in southern Brazil suggests that human cases of malaria of simian origin might be expected. Memórias do Instituto Oswaldo Cruz 79:461-463, 1984.

7. Ferreira MO, Rachou RG. Data on the incidence of Plasmodium malariae in the State of Santa Catarina. Revista Brasileira de Malariologia e Doenças Tropicais 18: 553-556, 1966.

8. Fundação Nacional de Saúde. Relatório Anual - Informes Estatísticos. Gerência Técnica de Malária/Coordenação de Controle de Doenças Transmitidas por Vetores/Departamento de Operações, Disponível em http://www.funasa.gov.br. Acesso em 20/set.2002, Brasília, DF, 2002.

9. Fundação Nacional de Saúde. Informe epidemiológico do Centro Nacional de Epidemiologia do Ministério da Saúde. Programa 
de Intensificação das Ações de Controle da Malária na Amazônia Legal 2002.

10. Marques AC, Gutierrez HC. Combate à malária no Brasil: evolução, situação atual e perspectivas. Revista da Sociedade Brasileira de Medicina Tropical 27 (supl III): 91-108, 1994.

11. Marques AC, Pinheiro EA. Fluxos de casos de malária no Brasil em 1980. Revista Brasileira de Malariologia e Medicina Tropical 34:1-31,1982.

12. Marques AC, Pinheiro EA, Souza AG. Um estudo sobre dispersão de casos de Malária no Brasil. Revista Brasileira de Malariologia e Doenças Tropicais 38: 51-75, 1986.
13. Passos ADC, Fialho RR. Malária: aspectos Epidemiológicos e de controle. Revista da Sociedade Brasileira de Medicina Tropical 31(supl II): 93-105, 1998.

14. Rachou RG, Ferreira MO. Kertezia as the group most responsible for malaria in the State of Santa Catarina. Revista Brasileira de Malariologia e Doenças Tropicais 18: 691-697, 1966.

15. Tauil PL. Malária: agrava-se o quadro da doença no Brasil. Ciência Hoje 2: 58-64, 1984

16. World Health Organization. Weekly Epidemiological Record 69:309-316, 1994. 